\title{
LOS PROPÓSITOS Y MÉTODOS DE INVESTIGACIÓN DE LA INCLUSIÓN EN EDUCACIÓN:
}

\section{ALGUNOS DESPLAZAMIENTOS}

THE PURPOSES AND RESEARCH METHODS OF INCLUSION IN EDUCATION: SOME DISPLACEMENTS

\section{Julián Eduardo Betancur Agudelo ${ }^{1}$}

Unidad Central del Valle del Cauca. Tuluá, Colombia.

\section{Napoleón Murcia Peña ${ }^{2}$}

Universidad de Caldas. Manizales, Colombia.

\section{RESUMEN}

El artículo presenta los resultados de un estudio bibliográfico que buscó definir las tendencias de investigación de la inclusión en educación, tomando en consideración los objetivos y métodos utilizados. Se realizó a partir de bases de datos científicas y tesis postgraduales nacionales e internacionales, con los siguientes descriptores (Inclusión Educativa; Educación Inclusiva). Se utilizaron unos focos de análisis,

1 Candidato a Doctor en Educación de la Universidad de Caldas, Magíster en Educación y Licenciado en Educación Básica con énfasis en Educación Física, Recreación y Deporte de la Universidad de Caldas, docente de planta tiempo completo de la Unidad Central del Valle del Cauca e Integrante de los grupos de investigación "Mundos Simbólicos: Estudios en Educación y Vida Cotidiana” y "Educación y Currículo" Tuluá, Valle del Cauca, Colombia. E-mail: jbetancur@uceva.edu.co. jbetancur@ uceva.edu.co julianbeta333@gmail.com Cvlac https://scienti.minciencias.gov.co/cvlac/visualizador/generarCurriculoCv.do?cod $\underline{r h=0000681725}$ Google scholar https://scholar.google.es/citations?user=oi63paUAAAAJ\&hl=es orcid.org/0000-0002-2890-826X 2 Posdoctor en narrativa y ciencia. Universidad Santo Tomás- Universidad de Córdoba. (2010-2012); Doctor en Ciencias sociales niñez y juventud. Universidad de Manizales - CINDE (2002-2006); Magister en Educación con énfasis en inclusión comunitaria. Universidad De Caldas - Pontificia Universidad Javeriana (1994-1996); Licenciado. Universidad Surcolombiana (1976). Profesor titular Universidad de Caldas, profesor invitado doctorados en Ciencias de la Educación de la Universidad del Magdalena, Universidad del Cauca. Profesor invitado doctorado en educación y derechos humanos, Universidad Surcolombiana, Profesor invitado en Doctorado en práctica pedagógica, Universidad Francisco de Paula Santander, Profesor doctorado en Educación Universidad de Caldas y líder de la línea Educación y vida cotidiana de este doctorado. Líder grupo Mundos simbólicos: estudios en Educación y vida cotidiana. napo2308@gmail.com napoleon.murcia@ucaldas.edu.co

Orcid https://orcid.org/0000-0001-9657-2086 Cvlac http://scienti.colciencias.gov.co:8081/cvlac/visualizador/generarCurriculoCv. do?cod_rh=0000070050 Google scholar https://scholar.google.es/citations? user=NGCAP8sAAAAJ\&hl=es 
tomados del estudio 'Regiones Investigativas de la Educación y Pedagogía en Colombia', a través de una matriz doxográfica. Los resultados muestran una gran relevancia hacia los métodos y objetivos direccionados a comprender e interpretar las realidades de la inclusión en educación.

PALABRAS CLAVE: Inclusión en Educación, Investigación, métodos, objetivos, enfoques, técnicas e instrumentos.

\section{ABSTRACT}

The article presents the results about a bibliographic study that sought to define research trends for inclusion in education, taking into consideration the objectives and methods used. It was made from scientific databases and national and international postgraduate thesis, with the following descriptions (educational inclusion; inclusive education). Analysis focuses were used, taken from the study 'Regiones Investigativas de la Educación y Pedagogía en Colombia', through a doxographic matrix. The results show great relevance to the methods and objectives aimed at understanding the realities of inclusion in education.

KEYWORDS: Inclusion in education, Research, Methods, Objectives, Approach, Techniques and measuring instrument.

\section{RESUMO}

$\mathrm{O}$ artigo apresenta os resultados de um estudo bibliográfico que buscou definir as tendências de pesquisa da inclusão na educação, levando em consideração os objetivos e métodos utilizados. Foi realizado a partir de bases de dados científicas e teses de pós-graduação nacionais e internacionais, com os seguintes descritores (Educational Inclusion; Inclusive Education). Foram utilizados alguns focos de análise, retirados do estudo 'Regiões de Pesquisa da Educação e da Pedagogia na Colômbia', por meio de uma matriz doxográfica. Os resultados mostram grande relevância para os métodos e objetivos que visam compreender e interpretar as realidades da inclusão na educação.

PALAVRAS-CHAVE: Inclusão na educação, Pesquisa, Métodos, Objetivos, Abordagem, Técnicas e instrumentos de medição.

\section{INTRODUCCIÓN}

Este artículo es una revisión de antecedentes de la tesis doctoral "Inclusión como Posibilidad de Reconocimiento", orientada en buscar -en las instituciones educativas- las realidades conversacionales de la inclusión. Se parte de considerar que la investigación es un constructo social y por tanto las dinámicas de su desarrollo cambian permanente en el marco de los imaginarios teóricos que los grupos de investigación proponen (Taylor, 2006); dicho esto, la forma de agrupar los hallazgos en este estudio se hacen siguiendo unas categorías generadas desde la investigación "Regiones educativas en educación y pedagogía en Colombia" (Ospina y Murcia, 2012), en el cual las categorías corresponden a un extenso trabajo de revisión de más de 1700 tesis de maestrías y doctorados que siguieron los procesos de codificación abierta, axial y selectiva definidos por Strauss y Corbin, (2002) en la construcción de teoría sustantiva.

Ciertamente, las comunidades académicas funcionan desde esas fuerzas que definen un "nosotros" y se funden en las prácticas sociales en las que les dan una especie de aval social; con esto, hacen que la práctica sea válida por los grupos sociales. Las agrupaciones que se consideran en el marco de la metodología planteada en este escrito, no son otra cosa que acuerdos validados socialmente, los cuales se han ido configurando desde las realidades conversacionales (Shotter, 1993) puesto que se generan formas prácticas de investigar sobre la inclusión. Justamente, los planteamientos ya descritos de Taylor (2006), respecto a los 
imaginarios teóricos, se podrían ubicar en esta misma racionalidad, debido a que son el trasfondo del acuerdo social de teorías que permiten generar realidades determinadas.

\section{METODOLOGÍA}

Hoyos propone los estados del arte como "investigaciones con desarrollo propio que se inscriben en el campo de la investigación documental. Sin embargo, su finalidad es dar cuenta de sentido sobre datos que apoyan un diagnóstico y un propósito en relación con el material sometido al análisis" (2000, pp.57). En relación con lo dicho, la recolección y procesamiento de la información sigue los métodos propios de los estudios documentales basados en el agrupamiento realizado por Murcia y Ospina (2012) que, además de proponer fichas para vaciar la información, genera categorías de análisis.

El modelo de agrupamiento antes mencionado es producto de un trabajo de investigación (tomado de tesis y maestrías en Colombia) que generó códigos y categorías emergentes que condensaron la información revisada Murcia y Ospina, 2012; Murcia, Murcia, 2018). Sin embargo, los autores realizaron adaptaciones a las agrupaciones en referencia, de acuerdo a las particularidades de la inclusión en Educación. La información procesada mediante el Atlas ti. 7.0 se caracterizó por la revisión de más de 70 investigaciones, en las cuales se analizaron unos focos de comprensión desde los propósitos, enfoques metodológicos y sus fundamentos epistemológicos.

Se propone inicialmente la red semántica y su respectiva descripción y análisis. Se considera que las categorías con altos niveles de relevancia social son las que aparecen en 20 estudios tomados como referencia; los niveles de relevancia medio se ubican entre 10 y 19 citas; los niveles de relevancia bajo, entre 3 y 9 citas, y los de niveles de relevancia social muy bajo entre 1 y 2 citas.

El modelo de ficha doxográfica utilizado fue:

Tabla 1:

Modelo de ficha Doxográfica. Fuente: (Murcia y Ospina, 2012, adaptado para este estudio).

\begin{tabular}{|c|l|c|c|c|c|}
\hline Titulo & Autores & $\begin{array}{c}\text { Año de } \\
\text { finalización }\end{array}$ & $\begin{array}{c}\text { Lugar de } \\
\text { investigación }\end{array}$ & $\begin{array}{c}\text { Objetivos - } \\
\text { Propósitos }\end{array}$ & $\begin{array}{c}\text { Enfoques y } \\
\text { Métodos }\end{array}$ \\
\hline & & & & & \\
\hline
\end{tabular}

\section{RESULTADOS}

Dadas las particularidades y el interés del artículo por analizar algunas tensiones que se muestran en los procesos de investigación en Educación e Inclusión se tomaran para este texto tres dimensiones: los objetivos, la metodología y los diseños, en consideración a esto se presentan las redes semánticas con sus descripciones buscando hacer el análisis de sus particularidades en la parte final del texto.

\subsection{Estado de objetivos, métodos y técnicas de la Investigación en Inclusión en Educación}

Los objetivos se agruparon desde la relación de sus formulaciones con verbos generalizados. Por otro lado, los métodos se agruparon desde su planteamiento explícito en el proyecto que, en la mayoría de los casos, no se propone como método sino como enfoque y, a su vez, incluye de manera directa las técnicas con base en los recursos utilizados para recolectar y procesar la información. 


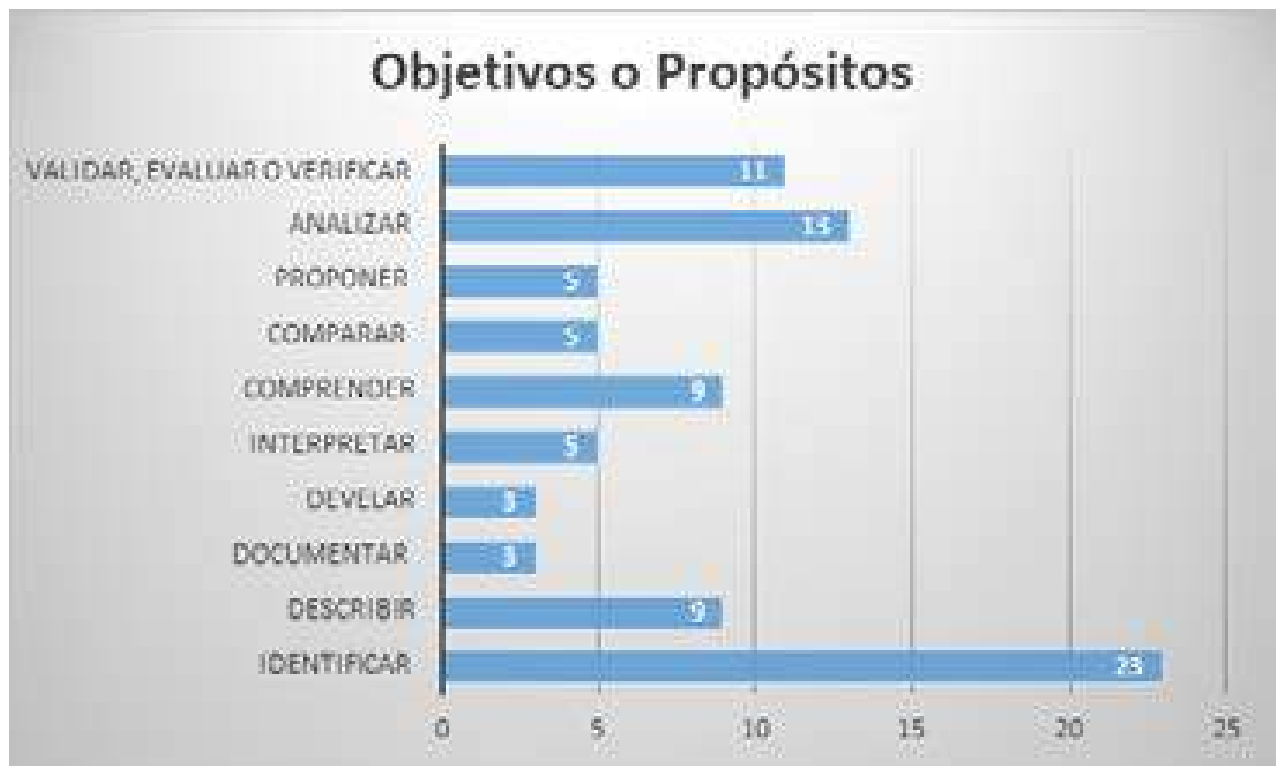

Gráfica 1. Red semántica Objetivos. Fuente: propia. (2019)

La mayoría de procesos de investigación se han dedicado a la identificación de cómo se llevan a cabo los procesos de inclusión en educación, con un nivel de relevancia social alto (23 citaciones). Al asociarse con los propósitos descriptivos (9), constituyen una relevancia social, ya que se relacionan entre sí y su interés está marcado en determinar cómo se llevan a cabo estos procesos.
En niveles de relevancia medio, se ubican objetivos que buscan analizar o verificar, (con 13 y 11 citaciones). En niveles bajos de relevancia social se ubican objetivos que buscan comprender, comparar y proponer (con 9, 5 y 5 citaciones). Entre tanto, en niveles que apenas son visibles se definen objetivos que pretenden documentar y develar las realidades de la inclusión en la Educación.

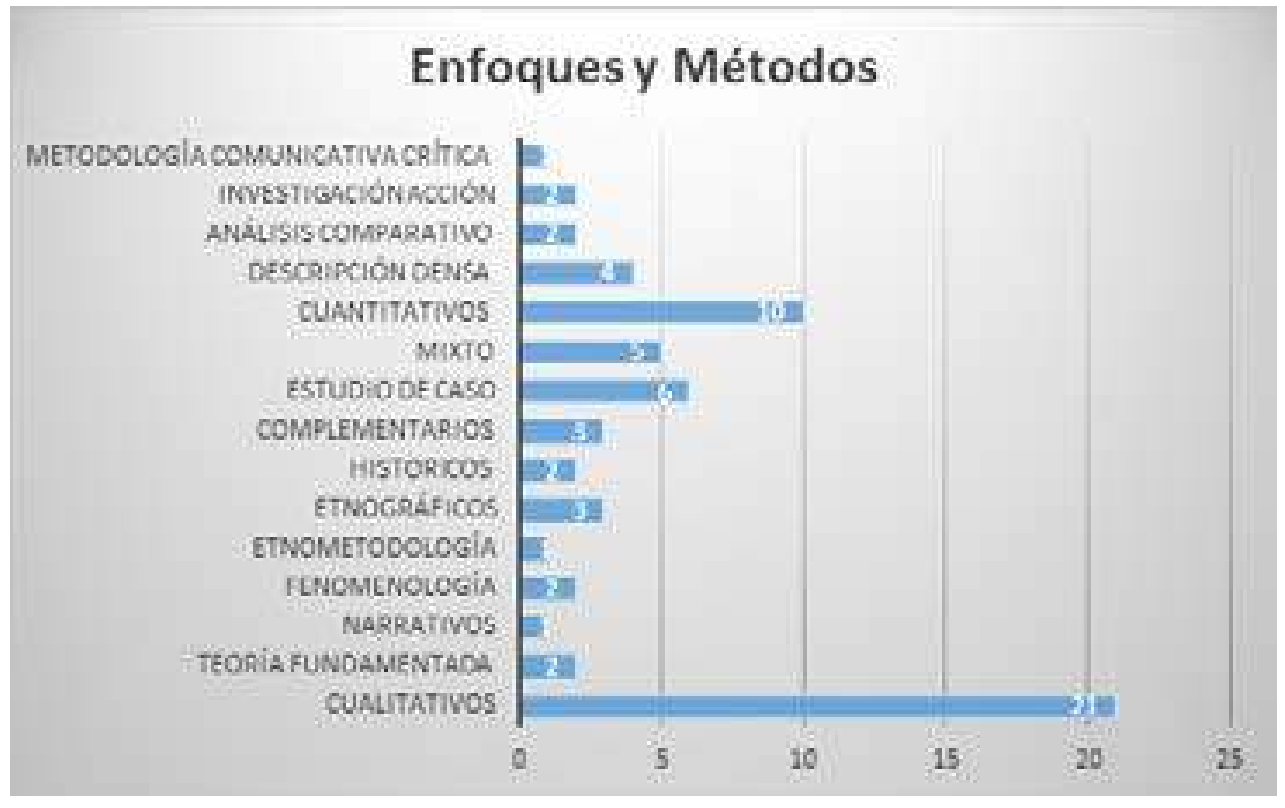

Gráfica 2. Red semántica opción Metodológica. Fuente: propia. (2019) 
En la gráfica es posible considerar que los métodos de mayor fuerza son los cualitativos, ya que la mayoría de los investigadores le han dado dicho enfoque (21), los cuales se realizan, en algunos casos, vinculando métodos desde la complementariedad (3), estudios de caso (6) o mixtos (5).

En segundo nivel de relevancia social, nivel medio, se ubican los enfoques cuantitativos (10), los cuales también se asocian con métodos mixtos, estudios de caso y enfoques de complementariedad. Con niveles de relevancia social bajo (entre 6, 5, 4 y 3 ) aparecen los estudios de caso, los métodos mixtos, los métodos de descripción densa y los métodos etnográficos y enfoques de complementariedad. Otros métodos que apenas emergen en las realidades conversacionales de los grupos analizados, aparecen en niveles muy bajos de citación, tales como, teoría fundamentada, fenomenología, análisis comparativos, investigación acción, históricos. Algunos apenas visibles son la metodología comunicativa crítica, etnometodología y los narrativos.

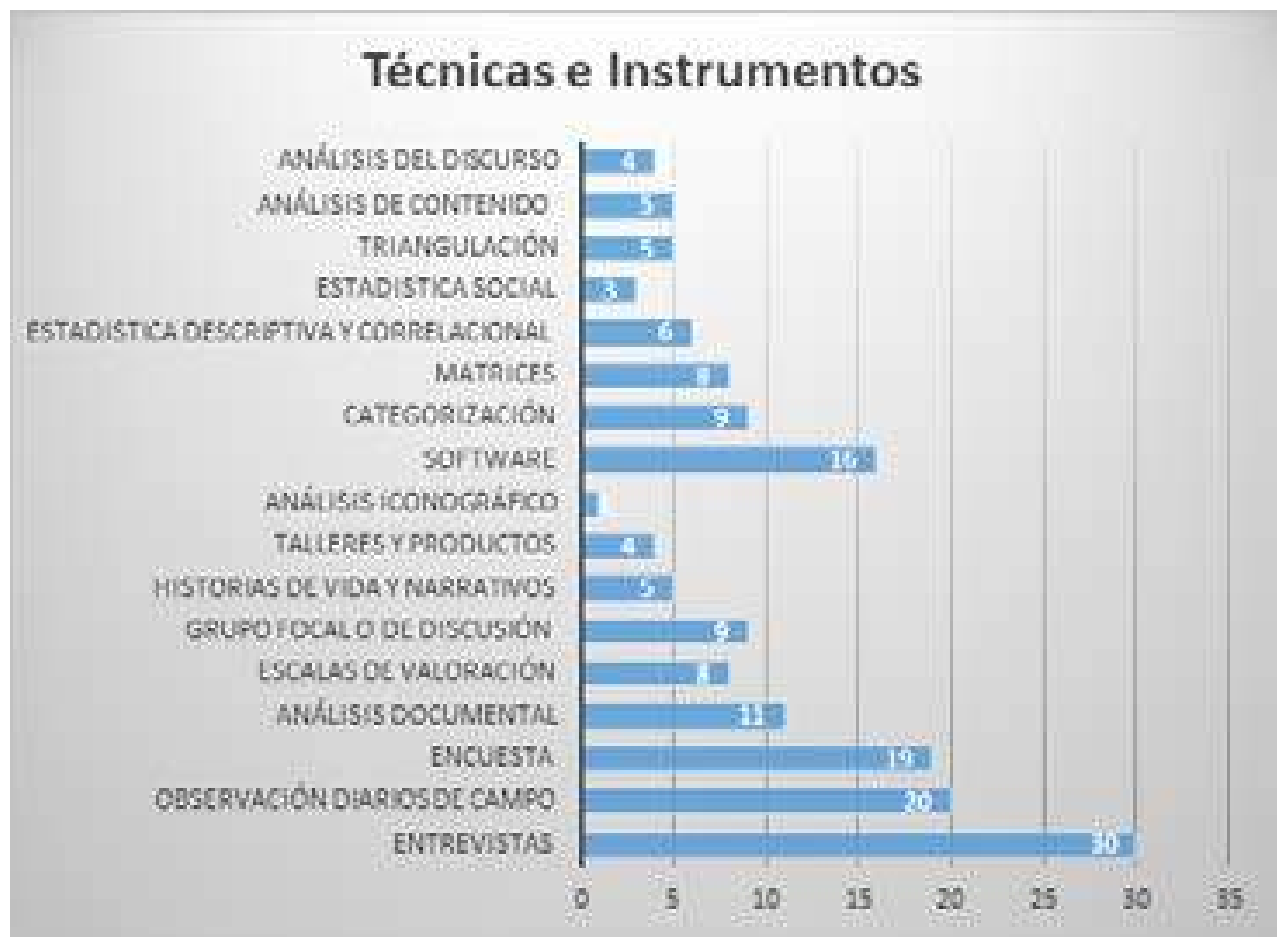

Gráfica 3. Red semántica técnicas e instrumentos. Fuente: propia. (2019)

Las técnicas e instrumentos utilizados para recolectar la información son muy variadas, siendo de mayor relevancia social las entrevistas (30), seguida por observaciones (20). Las encuestas, la aplicación de softwares educativos y el análisis documental (11), aparecen en niveles de relevancia medio (con 19, 16 y 11) respectivamente. Otras técnicas de recolección, procesamiento y análisis de la información, aparecen en niveles bajos de relevancia social, tales como la categorización, los grupos focales o de discusión (cada uno con 9) las escalas de valoración o las matrices de análisis (cada una con 8), la triangulación, las historias de vida o los análisis de contenido (con 5 cada una), los talleres y análisis de discurso (con 4 cada una), la estadística social (3) y el análisis iconográfico que aparece con una citación.

\section{ALGUNOS PUNTOS DE REFLEXIÓN}

\section{Y ANÁLISIS}

Al realizar un análisis de las direcciones que está tomando la investigación en educación sobre 
inclusión, nos lleva a considerar dos dimensiones que evocan la información recabada; la primera, referida a las relevancias y opacidades (Pintos y Galindo, 2003) o emergencias, y la segunda, referida a los desplazamientos que se generan.

En primer lugar, la mayoría de los estudios, desde sus descripciones, buscan identificar esos procesos y escenarios de inclusión, acudiendo a la exploración para identificar las características de las realidades, y la descripción para abordar sus componentes, con esto se acude a los niveles más básicos de la investigación (Cauas, 2019), no por esto, menos importante en la investigación social.

En la lógica de Michel Serres (1995) en esta categoría, se ubican aquellos estudios que paisajean las realidades con la intención de reconocer y definir los límites y características. Es interesante que son pocos estudios que trascienden estos niveles básicos hacia la reflexión sobre las categorías que configuran este campo. Estudios que buscan comprender, son poco explorados, y los propósitos transformacionales apenas se asoman en el mapa de la investigación en educación e inclusión.

Esta dirección está en contravía de las tendencias de la investigación en educación y pedagogía en Colombia, ya que los propósitos transformacionales y comprensivos han tomado en los últimos 10 años una gran relevancia social (Murcia y Murcia, 2018, Murcia y Ospina, 2012); a decir de los autores esta movilidad ha sido generada fundamentalmente por la inclusión de perspectivas sociocríticas definidas en las maestrías y doctorados generados en la última década en Colombia.

No obstante, existe una tendencia predominante de enfoques cualitativos y entrevistas como técnica con enorme relevancia social en la recolección de información. Claro está, hay que reconocer que estos pueden quedarse en niveles descriptivos de investigación, si no se realiza bien la entrevista. De hecho, los enfoques como la etnografía, la teoría fundada, la complementariedad, los enfoques narrativos, históricos, análisis de discurso o iconográficos, que podrían dar cabida a niveles más profundos de comprensión y explicación no causal de las realidades de la inclusión en Educación, son apenas incipientes en los estudios referentes. Sin realizar procesos de comprensión de estas dinámicas sociales, es difícil generar procesos efectivos de transformación, puesto que ellos requieren niveles fuertes de empoderamiento social que impliquen modificación de las prácticas sociales de inclusión en educación tal y como lo menciona Slee (2011).

El estudio de la investigación social, y en particular en Educación en inclusión, es más que utilizar un método, dado que la naturaleza compleja de estos fenómenos implica esculcar en recónditas profundidades de los problemas, abordada desde su naturaleza social, para comprender lo que se puede considerar como la primera creación del investigador, y así llegar al objeto discursivo problematizado. Ese objeto se configura desde el análisis de las regularidades sociales presentes o ausentes en las realidades conversacionales de los contextos próximos y lejanos de justificación y desarrollo, $y$ se constituye en el primer constructo del investigador. Estos objetos no aparecen del todo predefinidos ante los ojos del investigador, sino que se muestran desde fragmentos, como códigos o categorías en las realidades sociales, que deben ser descifradas por el investigador, para así generar su construcción.

Siguiendo a Foucault (2002), los objetos discursivos son construcciones sociales que se consolidan desde los enunciados que las personas realizan en diferentes contextos y tiempos. Por tanto, son construcciones socio/ históricas, puesto que se evidencian en diversas superficies de emergencia. Como tal, ellos están 
sujetos a unas condiciones socio/históricas que hacen posible su aparición. De modo que, no aparece un solo método de investigación, pues, estos no son claramente definidos como tal, mientras que los diseños son previstos desde técnicas e instrumentos de recolección, e incluso de procesamiento de la información.

Los investigadores configuran sus objetos discursivos desde el reconocimiento progresivo que hacen en sus visitas a las realidades conversacionales, desde múltiples y variadas interacciones que en ellas van logrando. Desde estas configuraciones definen las formas de abordar esos objetos discursivos y sus fenómenos; ellas pueden ser muy variadas, algunas siguen lógicas convencionales, utilizando métodos cualitativos y cuantitativos y formas que van desde enfoques de complementariedad hasta análisis iconográfico.

Con lo expuesto, no se puede negar que la investigación en el campo de la inclusión en educación es un constructo social que se va configurando en la medida de sus proximidades e intereses de investigación, ya que no existen métodos únicos para realizarla, pues estos son creados de acuerdo a la comprensión de la naturaleza de los objetos discursivos y los problemas; de hecho algunas perspectivas construccionistas de inclusión la asumen como una posibilidad de reconocer al otro que había sido excluido en su condición de humanidad (Honneth, (2007), lo cual es una abierta o profunda postura hacia la construcción social de la inclusión (Betancur, 2016).

En segundo lugar, en la lógica convencional, los métodos deberían corresponder a los objetivos de investigación, en el entendido que el método es el camino para resolver el problema, por tanto, en esta perspectiva, lo más importante para resolverlos sería el haber definido el método. Pero, lo que muestran los resultados de la revisión documental es que los investigadores no hacen mucho énfasis en el método que se va a utilizar, categoría que, generalmente, se ve desplazada por la consideración de enfoque. Esto deviene en una apertura epistemológica de la investigación, en la que se amplían los marcos estructurados a nuevas posibilidades de entender y comprender la realidad social sin estar centrados en la importancia de dicha comprensión (Murcia y Jaramillo, 2008)

En la gran mayoría de los estudios consultados, el enfoque es cualitativo, en consideración a la clasificación utilizada por algunos teóricos de la investigación Yánez (2019), Hernández, Fernández y Baptista (1998), entre otros, para quienes los enfoques de investigación pueden ser cualitativos o cuantitativos. Ahora bien, solo por tomar un ejemplo de lo consultado en su trabajo, Moreno, Moreno, y Amaya, (2015) explicitan: ““El presente estudio se realizó con enfoque cualitativo, dado que su visión holística, humanista y reflexiva permite tener una perspectiva total del objeto de estudio"' además, los investigadores quieren mostrar la relación de ese enfoque con su intencionalidad comprensiva y exponen: "En ese sentido, se pretende comprender e interpretar las prácticas pedagógicas de los docentes que atienden niños con necesidades educativas especiales en los colegios Café Madrid, Colegio Rafael García Herreros e Instituto Promoción Social del Norte de las comunas 1 y 2 de Bucaramanga (pp.38).

Habría que decir también que, en el estudio referido por Rosa y Villar, $(2015,12)$ se asume al enfoque cuantitativo desde las consideraciones mismas de la operacionalización del problema, en un estudio sobre la importancia de las actitudes hacia la inclusión e interculturalidad “"según variables sociodemográficas edad, género, experiencia en interculturalidad y/o discapacidad, título universitario y ciclo estudios"'.

Miremos los dos casos planteados; en el primer caso el objetivo se define desde la fuerza del enfoque, "comprender", pese a ello, no todos 
los enfoques cualitativos son comprensivos. De hecho, Hernández Fernández y Baptista (1998) aseguran que lo cualitativo se relaciona con el tipo de variables que se van a operacionalizar, así estas sean procesadas desde métodos cuantitativos o estadísticos. Es así como, un enfoque cualitativo no necesariamente buscaría comprender la realidad sino explicarla en la lógica del dominio de variables, o solamente describirla, con lo cual estaría de acuerdo el segundo ejemplo tomado como referencia.

Quizá por esta razón la relevancia social de los objetivos en los estudios consultados, no tiene correspondencia con los métodos más utilizados, pues mientras los objetivos destacados son descriptivos, los métodos (enfoques en la mayoría de los estudios consultados) más importantes son cualitativos. ¿Se asume acaso que los enfoques cualitativos buscan identificar, describir o analizar una realidad?, de ser así, ¿Por qué en algunos estudios asumidos desde enfoques cualitativos se plantea, explícitamente, comprender las realidades?

Al parecer, para los investigadores, no es muy relevante la coherencia entre los objetivos y el método o enfoque de investigación, lo cual genera aún mayor incertidumbre frente a la validez ecológica que algunos investigadores definen y que se sigue en la mayoría de las exigencias institucionales, por lo menos en Colombia $^{3}$. Veamos otro caso:

El enfoque mixto que orientó el diseño de la investigación permitió que se realizará una aproximación con pretensión objetivista a través de la aplicación del Índice de Inclusión propuesto por el Mineducación y, una con pretensión no comprensiva en la que se intentó recuperar la historia vivida por los actores educativos y la manera como han constituido sentido de las experiencias, a través de la realización de grupos focales con padres, docentes,

3 En la mayoría de los criterios de evaluación de tesis doctorales y de maestrías, se exige coherencia entre problema, objetivos y el método utilizado. estudiantes y directivos, en tres instituciones educativas que reportaron en el Índice de tres categorías: prácticas, políticas y cultura escolar (Loaiza, 2011, 167).

Entonces, por una parte, se asume un enfoque que articula métodos cualitativos y cuantitativos de investigación, pero, por otro lado, no es muy importante si el enfoque permite comprender en profundidad la realidad o explicarla desde el dominio objetivista de ella.

\section{En un caso analizado se evidencia el siguiente aparte:}

Esta investigación es de tipo transversal con un enfoque cualitativo. Como estrategias de recolección de información se utilizaron: el análisis documental, la entrevista individual, la observación no participante, el grupo focal, y encuestas de caracterización para estudiantes, docentes y padres de familia. Se empleó un diseño de investigación acción participativa (IAP). (Beltrán, Martínez, y Torrado, 2015, pág. 62).

Evidentemente, los investigadores no definen su enfoque ni el método de investigación, o por lo menos las descripciones no corresponden a la naturaleza originaria de los métodos utilizados. Varias incoherencias se muestran en el aparte, por un lado, en la base de la IAP, (la cual es el diagnóstico social que se realiza por parte de los líderes naturales), y por otro, el análisis documental no es esencial en la IAP. (Fals, 1997).

Lo expuesto muestra que, para una gran mayoría de los investigadores tomados como referencia y que asumen como enfoque lo cualitativo o cuantitativo, no es importante la coherencia que pueda existir entre el enfoque definido y los objetivos propuestos, o por lo menos, no existe una relación fuerte y directa entre el enfoque y el propósito de la investigación. Quizá, esto esté relacionado con la ambigüedad misma del planteamiento de lo cualitativo, pues como foco 
implicaría fraccionar el mundo en cualidades no medibles, sin embargo, en muchos casos, se utilizan métodos estadísticos para procesar esas cualidades aparentemente "'no medibles"”. ¿Lo cualitativo es por las cualidades o por el método de procesamiento? pues si utilizo variables cualitativas y las mido desde la estadística, estaría utilizando un método cualitativo en la consideración teórica del mundo pero, a la vez, un método cuantitativo en la consideración metodológica de acceso a esa realidad del mundo, lo cual es, en sí mismo, una incoherencia epistemológica.

Ahora, entender la inclusión en educación desde las incoherencias epistemológicas tiene una fuerte relación con las diferentes formas en la que se ha reducido en el escenario escolar, puesto que, se logra evidenciar se encuentra una tendencia muy marcada en el cumplimiento de indicadores y procesos con respecto a la calidad educativa (Booth y Ainscow, 2006). También, es notorio el diagnostico de las realidades sociales sobre las percepciones que tienen los miembros de la comunidad educativa y sus dificultades en el proceso académico (Ortiz, 2016), lo termina generando las pocas transformaciones en los sistemas educativos, lo cual sigue prolongando procesos de exclusión a pesar de su misión incluyente (Soto, 2007). Todo esto es un ejemplo claro que no se están generando procesos de comprensión profunda de los actores que se pretenden incluir.

Pese a esta prevalencia social, hay emergencias metodológicas como los enfoques complementarios, los enfoques narrativos, etnográficos y los crítico sociales (ya enumeradas), que configuran sistemas de acceso a las realidades, de acuerdo a la naturaleza próxima de los objetos discursivos y a los intereses del investigador que se salen de las racionalidades hegemónicas de los enfoques cualitativos y cuantitativos.
Positivamente, varios estudios tomados como fuente de información, asumen como relevante el enfoque antes que el método de investigación; esto es, visto desde una perspectiva en la cual se abordan las temáticas a investigar y que corresponde fundamentalmente al interés del problema; por tanto, el enfoque está estrechamente ligado a la naturaleza del objeto discursivo configurado. En ellos, el investigador, realiza un proceso de aproximación a la realidad para poder descifrar desde este aquellos focos que se deben relevar. En un estudio realizado por Betancur (2013) los focos que se deberían investigar en los procesos de inclusión en la clase de educación física fueron definidos desde la aproximación a la realidad de la clase y no desde las teorías formales definidas en este sentido. (Pintos, 2001, 2003).

Esto, por cuanto los enfoques emergentes buscan en la complejidad de las realidades mismas, como también, en las categorías de los fenómenos que van a ser profundizados y, por tanto, se terminan alejando de la racionalidad cualitativa o cuantitativa y su dualismo ontológico; en ellos las categorías de la realidad surgen de la realidad misma y logran su validez a partir de la triangulación entre actores sociales y teorías sustantivas sobre realidades similares (Murcia y Jaramillo 2008; Orrego y Pórtela, 2009; Delgado y Blanco, 2015; Betancur, 2013; Cárdenas, 2014; Vera, Palacio, y Patiño, 2014; Mosquera, 2011; Breitenbach, Honnef, y Costas, 2016; Domínguez, 2009).

Un tercer aspecto a considerar en la relación entre estos tres componentes de las investigaciones en Educación en Inclusión, es el papel del diseño y las técnicas e instrumentos que lo definen. Tal y como se ha mostrado, para la mayoría de los estudios no es relevante hablar sobre el método de investigación seguido, en cambio, se da importancia al enfoque. Pero, lo que define el proceso a seguir en la recolección, análisis e interpretación de la información está 
definido por el diseño, que se configura desde las técnicas e instrumentos utilizados.

Esta perspectiva podría ser analizada desde dos consideraciones; la primera en consideración a que la realidad, "'En su sentido más general, diseño es una forma de política: la humanidad luchando por darle forma a su entorno $y$ su sociedad, de manera de satisfacer sus necesidades"' (Serrano, 2001, pp.3), lo cual deja sentado el hecho que diseñar una investigación social, es un compromiso ético-político, que responde a un acto creativo del investigador que busca una forma de acceder, procesar e interpretar la información de un fenómeno social y humano, desde una mirada del mundo, del ser humano y sus relaciones. Esa mirada desde la cual se asume el mundo, empuja a configurar un objeto discursivo determinado $y$, por consiguiente, a crear una forma particular para acceder a dicho objeto configurado.

De manera que, es en verdad el segundo gran proceso de creación del investigador, pues implica analizar las particularidades del objeto discursivo en sus múltiples dimensiones $\mathrm{y}$ cruzarlas con los intereses del investigador para desde ahí proponer una forma científicamente, para acceder al problema a investigar. O sea, en definitiva, la relación entre la forma dada al objeto discursivo y el diseño de investigación, es lo que le da el valor epistemológico a lo encontrado en la investigación.

La segunda opción de analizar la preponderancia que dan los investigadores al enfoque sobre el método podría dar a entender el excesivo interés en lo meramente instrumental; sin embargo, si las técnicas e instrumentos están definidas estrechamente desde la naturaleza del objeto discursivo de investigación, se puede argumentar una coherencia elevada; no así, si el problema es adaptado a un método universal.

Dos asuntos son importantes de considerar cuando se analizan los diseños, confrontados con los objetivos y los métodos; uno de ellos es la estrecha relación que, en general, dejan ver estudios tomados como referencia, entre los objetivos y las técnicas e instrumentos utilizados, (recordemos que los objetivos más utilizados son aquellos que buscan identificar y describir), lo cual coincide con las técnicas de mayor relevancia: la Observación y la Entrevista, es decir, los enfoques que se han denominado cualitativos. Cosa que no sucede con los enfoques cuantitativos, pese a ocupar un sitial de gran importancia.

Sólo por tomar algún caso presentamos este fragmento:

El siguiente documento tuvo como objetivo hacer visible la situación de vulnerabilidad que sufren los niños expuestos a la violencia de género dentro de la familia [...]. Se realizaron entrevistas, se examinaron los registros y se obtuvo información del cuestionario Screening Index de Barcelona Childhood antes y después (exactamente tres meses después) de que se completara la intervención. El propósito de dicha investigación fue determinar el nivel de experimentación y desarrollo de los síntomas del trastorno de estrés postraumático (TEPT), así como los factores que han influido en el proceso de afectación. (Riquelme et al. 2019). (pp. 113)

La gran mayoría de los estudios analizados, enfatiza en las técnicas de recolección de la información, caso de los estudios de Garnique (2012) y Krichesky, (2014), quienes utilizan como fuente principal la entrevista, las investigaciones de Vásquez, Méndez, y Mendoza (2015), Castro (2015), que toman la observación como técnica y el diario de campo como instrumento de recolección; los trabajos realizados por Sanhueza, Granada, y Bravo, (2012); Montánchez (2014), quienes utilizan la encuesta; los procesos llevados a cabo por Ramírez (2014), Vrecer, Javrh, y 
Capllonch (2010), que acuden al análisis de documentos institucionales; las investigaciones de Engelbrecht, Nel, Nel, y Tlale, (2015) que trabajan con grupos focales o de discusión; estudios como los desarrollados por López (2014) utilizaron las escalas de valoración, como las tipo Likert; o las investigaciones de Bocanegra (2008), quien utilizó las historias de vida y las narrativas; o los estudios de Maldonado, Ortiz, y Giraldo (2012); Llorent y López (2013); quienes acuden a los talleres y dibujos.

En muchos casos los investigadores se preocupan por definir los instrumentos de procesamiento, como en el caso muñoz, López, y Assaél, (2015) (Ohajunwa, Mckenzie, Hardy, y Lorenzo, 2014) quienes utilizan softwares como el Atlas-ti y el SPSS ${ }^{4}$. En otros se muestra el tipo de análisis a que acudieron para resaltar el procesamiento comprensivo, "procesos de codificación y categorización" (BeltránVillamizar, Martínez-Fuentes, y Vargas-Beltrán, 2015; Barbosa-Vioto y Vitaliano, 2013; Betancur, 2013; Vera, Palacio, y Patiño 2014).

Esta relevancia también se puede apreciar en trabajos que, además de plantear el método, acuden al diseño para mostrar la relación con los objetivos propuestos:

El principal objetivo de este trabajo de investigación es reflexionar sobre la manera de fomentar la inclusión social a través de los lenguajes artísticos. En concreto, la finalidad es escuchar y recoger las opiniones de los profesionales que trabajan para promover la inclusión social desde los Centros Abiertos (Centros de Atención Primaria de Servicios Sociales en Cataluña). La investigación que se presenta es fruto de un estudio mixto que combina la estrategia cualitativa de la entrevista grupal

$4 \quad$ Programa que aborda el proceso analítico: planificación, colección de datos, análisis, presentación de informes e implementación de datos cualitativos desarrollado por la IBM. y el instrumento del cuestionario desde la perspectiva cuantitativa, en el que han participado 97 profesionales de Centros Abiertos de Cataluña. (Mundet, Fuentes y Crescencia, 2017). (pp. 143)

En todos los casos la relevancia que los investigadores le otorgan a las técnicas, hace prever que, en este campo de estudio, el interés por los diseños supera el de los métodos, pero que, además, los objetivos corresponden casi estrictamente al diseño de investigación y ocasionalmente al método definido; lo cual se presenta como una contradicción si se relaciona estos resultados con los avances reales de la inclusión en Colombia, ya mencionados; puesto que, al definir los diseños desde los problemas reales se podría tener una mejor comprensión de las dinámicas sociales de la inclusión; sin embargo, dado que la mayoría de las técnicas e instrumentos utilizadas favorecen la descripción sobre la comprensión de las realidades de la inclusión, las investigaciones no están aportando los suficientes elementos que permitan comprender las dinámicas profundas de los procesos incluyentes en educación.

\section{ALGUNAS APERTURAS}

En primer lugar, el análisis de los estudios sobre inclusión en educación hace un énfasis muy fuerte sobre el enfoque y diseño antes que el método de investigación, lo cual es positivo ante los diseños corresponden a la naturaleza misma de los objetos de enunciación; pese a ello, cuando se hace un análisis tangencial de las realidades de la práctica de la inclusión en Colombia, se evidencia que no hay transformaciones profundas en la forma de asumirla (Betancur, 2020), lo cual indica que no basta con investigar las realidades de la inclusión en educación, es importante recabar en sus dinámicas para poder comprenderlas y generar, desde su análisis, transformaciones, tanto en la manera de decir y representarla, así como también en el modo de 
hacer y ser como sujetos incluyentes (Murcia, 2011).

La lógica de (Shotter,1993) que empuja los análisis de estas dinámicas, desde las realidades conversacionales, es una apuesta que podría darnos algunos elementos de gran valía para los estudios de la inclusión en los procesos educativos; pues ella permite re-conocer y reconocer-nos como sujetos de inclusión, desde los acuerdos logrados en el bullicio de la vida cotidiana. Es otorgando el valor a la comprensión de lo que está pasando que se logran cambio profundos tanto en la construcción de la teoría sobre la inclusión como en las formas de ser/ hacer en ella.

En segundo lugar, este desplazamiento (de los enfoques sobre los métodos), muestra que no es el método lo más importante para solucionar el problema, sino la comprensión del objeto discursivo y la creación de un diseño que corresponda con este objeto creado. Por tanto, el diseño puede contener varios métodos o, simplemente no presentarlos tácitamente, ya que, lo fundamental es que cuenten con un ordenamiento de técnicas e instrumentos que ayuden al investigador a resolver los problemas de acceso a la información y, a su vez, facilitar el procesamiento y la interpretación, acorde con naturaleza del problema. Este desplazamiento obliga a repensar las teorías de investigación en este campo y, en general, la investigación social que aún propone la prioridad del método.

No obstante, la prevalencia de los diseños sobre los métodos, quienes asumen lo cualitativo lo hacen tomando esta premisa como enfoque, lo cual no limita los estudios a la consideración de variables operacionales de un método Hernández, Fernández y Baptista (1998), sino que lo convocan desde las formas de asumir las realidades, de contemplar el mundo social y humano; desde esas dinámicas que lo hacen complejo y que son impensables desde las racionalidades de la medición cuantitativa. Porque:

El proceso de investigar de manera cualitativa presenta un reto, porque los procedimientos para organizar las imágenes no están bien definidos y se basan en procesos de inferencia y comprensión. El laboratorio de la investigación cualitativa es la vida cotidiana y a esta no puede metérsele en un tubo de ensayo, prenderla, apagarla, manipularla. El desarrollo, la descripción y la operación de la teoría suelen ser resultado y producto del proceso de investigación, más que medios y herramientas empleados al realizar la investigación (MORSE, 2003:4-5)

Lo anterior, lleva a considerar que a la investigación en inclusión y educación le asiste la fuerza de la construcción social, toda vez que sus métodos y diseños dependen de las perspectivas o imaginarios teóricos que se hayan ido configurando en las realidades conversacionales de los grupos de investigación.

\section{REFERENCIAS BIBLIOGRÁFICAS}

Allard-Serrano, José Manuel. ¿Diseño como disciplina? Editorial ARQ, (49), pp. 2-4. (2001). Chile https://scielo. conicyt.cl/scielo.php?script=sci_artte xt\&pid=S0717-69962001004900002 (Consultado el 14 de marzo de 2018)

Amores-Fernández, Francisco Javier, y RitaccoReal, Maximiliano. "Estudiantes en riesgo de exclusión educativa en secundaria. Percepciones del profesorado implicado en programas extraordinarios de prevención del fracaso escolar". Enseñanza $y$ Teaching: Revista Interuniversitaria de Didáctica. 34(1):137. (2016) https://www.researchgate.net/ 
publication/303801066 Estudiantes en_riesgo_de_exclusion_educativa en_secundaria_Percepciones. del_profesorado_implicado_en programas_extraordinarios_de prevencion_del_fracaso_escolar (Consultado el 25 de Marzo de 2018)

Amores-Fernández, Francisco Javier, y RitaccoReal, Maximiliano. "Buenas Prácticas Educativas en Centro Escolares ubicados en Zonas de Riesgo de Exclusión Social". Editorial Pulso. 69-88. (2011). España. https://dialnet.unirioja. es/servlet/articulo?codigo $=3793712$ (consultado el 14 de Marzo de 2018)

Barbosa, Josiane y Vitaliano, Celia. Educação Inclusiva e Formação Docente: Percepções de Formandos em Pedagogia. Magis. Revista Internacional de Investigación en Educación, 353-373. 2013. Colombia https://dialnet.unirioja. es/servlet/articulo?codigo $=4773624$ (consultado el 18 de marzo de 2018)

Barca, Alfonso Mascarenhas, Suely-Aparecida, Brenlla, Juan Carlos, Porto, Ana María, y Barca, Eduardo. "Motivación y Aprendizaje en el Alumnado de Educación Secundaria y Rendimiento Académico: un Análisis desde la Diversidad e Inclusión Educativa". Revista Amazónica, 9-57. (2008). Brasil https://dialnet.unirioja.es/servlet/ articulo?codigo $=4030112$ (Consultado el 20 de marzo de 2018)

Beltrán-Villamizar, Yolima Ivonne, MartínezFuentes, Yexica Lizeth, y TorradoDuarte, Omar Elías. "Creación de una comunidad de aprendizaje: una experiencia de educación inclusiva en Colombia". Revista Encuentros, 5772. 2015. Colombia http://www.scielo. org.co/pdf/encu/v13n2/v13n2a04.pdf (Consultado el 16 de Marzo de 2018)

Beltrán-Villamizar, Yolima Ivonne., MartínezFuentes, Yexica Lizeth, Vargas-Beltrán,
Ángela Sofía. "El Sistema Educativo Colombiano en el Camino hacia la Inclusión. Avances y Retos". Editorial Educación y Educadores, 62-75. (2015). Colombia https://dialnet.unirioja. es/servlet/articulo?codigo $=5429700$ (Consultado el 17 de Marzo de 2018)

Berruezo-Adelantado, Pedro Pablo. "Educación Inclusiva en las Escuelas Canadienses. Una Mirada desde la Perspectiva Española". Revista Interuniversitaria de Formación del Profesorado, 179-207. 2006. España https://www.redalyc.org/ pdf/274/27411341012.pdf (Consultado el 12 de Marzo de 2018)

Betancur-Agudelo, Julián Eduardo. "Prácticas Incluyentes y Excluyentes en la Clase de Educación Física". Revista Educación Física y Deporte, 1433- 1440. 2013. Colombia https://revistas.udea.edu. co/index.php/educacionfisicaydeporte/ article/view/17893 (Consultado el 14 de Marzo de 2018)

Betancur-Agudelo, Julián Eduardo. "Una Inclusión Excluyente: Reconfigurando la Inclusión en la Escuela." Revista de Investigaciones, 178-188. (2016). Colombia http://www.revistas.ucm.edu. co/ojs/index.php/revista/article/view/69 (Consultado el 20 de Marzo de 2018)

Betancur-Agudelo, Julián Eduardo. "La inclusión como posibilidad de reconocimiento. Tesis doctoral en evaluación". Doctorado en Educación. Universidad de Caldas. Colombia. (2020)

Bocanegra-Acosta, Elsa María. "Del Encierro al Paraíso. Imaginarios Dominantes en la Escuela Colombiana Contemporánea: una Mirada desde las Escuelas de Bogotá". Revista Latinoamericana de Ciencias Sociales, Niñez y Juventud, 319-346. 2008. Colombia http:// biblioteca.clacso.org.ar/Colombia/ alianza-cindeumz/20130710073003/ ArtElsaMariaBocanegraAcosta.pdf 
(Consultado el 10 de Abril de 2018)

Booth, Tony y Ainscow Mel. "INDICE DE INCLUSIÓN: Desarrollando el aprendizaje y la participación en las escuelas". CSIE: Mark Vaughan. (2006) https://www.eenet.org.uk/resources/ docs/Index $\% 20$ Spanish $\% 20$ South $\% 20$ America\%20.pdf

Bravo-Cóppola, Laura Inés. "Percepciones y Opiniones hacia la Educación Inclusiva del profesorado y de las/os equipos directivos de los centros educativos de la dirección regional de enseñanza de Cartago en Costa Rica". 2013. Alicante, España: Tesis Doctorales Teseo.

Breitenbach, Fabiane Vanessa, Honnef, Cláucia, y Tonetto-Costas, Fabiane Adela. "Educação inclusiva: as implicações das traduções e das interpretações da Declaração de Salamanca no Brasil". Ensaio: Avaliação e Políticas Públicas em Educação, 359-379. 2016. Brasil https://www.scielo.br/pdf/ensaio/ v24n91/1809-4465-ensaio-24-91-0359. pdf (Consultado el 27 de Marzo de 2018)

Calvo, Gloria y Camargo, Marina. "Dos Propuestas para la Inclusión de los Jóvenes en el Sistema Educativo." PerspectivaEducacional, 147-166.2013. http://www.perspectivaeducacional.cl/ index.php/peducacional/article/view/93 (Consultado el 16 de Marzo de 2018)

Campomar, Gloria del Carmen, Crescente Stella, Tuñón Lanina y Soledad Noblega. "Reflexiones en Torno al Proceso de Implementación de un Programa Social en el Campo de la Actividad Física en Poblaciones Vulnerables. Aportes para la Formación del Profesorado de Educación Física y la mejora de las Políticas Públicas". Educación Física y Ciencia. Vol. 15, No 2. Facultad de Humanidades y Ciencias de la Educación. (2013). http:// sedici.unlp.edu.ar/handle/10915/34085
(Consultado el 13 de Marzo de 2018) Cárdenas, Yenny Alexandra. "El Veredicto Docente: Incidencia de los Juicios de Valor en los Esquemas de Autovaloración de los Escolares". Manizales, Caldas, Colombia: Tesis Programa de Antropología. (2014).

Cardona-López, Marta, Loaiza de la Pava, Julián, y Ospina-Serna, Héctor Fabio. "Exclusión e Inclusión: lectura de narrativas de un grupo de jóvenes constructores de paz". Revista Colombiana de Educación, 142-168. (2008). https://revistas.pedagogica.edu. co/index.php/RCE/article/view/5284 (Consultado el 15 de Marzo de 2018)

Castro-Cáceres, Ricardo Andrés. "Imaginarios Sociales Instituyentes e Instituidos sobre la Educación Inclusiva de Profesores que conforman Equipos de Aula de Colegios con Programas de Integración Escolar en la Comuna de San Pedro de la Paz". Santiago de Chile: Universidad Academia de Humanismo Cristiano. (2015).

Cauas, Daniel. "Definición de las variables, enfoques y tipo de investigación". Bogotá: Biblioteca electrónica Universitaria. (2015).

https://scholar.google.com/ scholar?hl=es\&assdt=0\%2C5\&q=nivelt descriptivo+de+investigaci $\%$ C3\%B3n\&oq=ni

(Consultado el 15 de Marzo de 2020)

Delgado-Sanoja, Hellman, y Blanco-Gómez, Gisela. "Acercando la Brecha entre la Equidad y la Igualdad en las Oportunidades. La Inclusión Educativa". Revista Nacional e Internacional de Educación Inclusiva, 67-88. (2015). https://revistaeducacioninclusiva.es/ index.php/REl/article/viewFile/91/88 (Consultado el 18 de Marzo de 2018)

Diez, Adriana Cecilia. Las "Necesidades Educativas Especiales". Políticas 
Educativas en torno a la Alteridad. Cuadernos de Antropología Social, 157-171. (2004). https://core.ac.uk/ download/pdf/230946704.pdf (Consultado el 19 de Marzo de 2020)

Domínguez, Antonio lañez. Prisioneros del Cuerpo "La Construcción Social de la Diversidad Funcional". España: Diversitas Ediciones. (2009).

Domínguez-Alonso, José, López Castedo, Antonio, y Vásquez-Varela, Elia. "Atención a la Diversidad en la Educación Secundaria Obligatoria: Análisis desde la Inspección Educativa". Aula Abierta, 70-76. (2016). https:// www.sciencedirect.com/science/article/ pii/S0210277316300026 (Consultado el 21 de Marzo de 2018)

Duk-Homad, Cinthia, y Narvarte-Eguiluz, Libe. "Evaluar la Calidad de la Respuesta de la Escuela a la Diversidad de Necesidades Educativas de los Estudiantes". Revista Electrónica Iberoamericana sobre Calidad, Eficacia y Cambio en Educación, 137-156. (2008). https:// revistas.uam.es/index.php/reice/article/ view/5446 (Consultado el 14 de Marzo de 2018)

Elichiry, Nora Emilce, Arrúe Carola, Aizencang Noemi, Maddonni Patricia, Nakache Débora, y Scavino, Carolina. "Sistemas de Aprendizaje e Inclusión Educativa”. Procesos Cognitivos e Interactividad. XII Anuario de Investigaciones, 5358. (2004). https://www.redalyc. org/pdf/3691/369139941004.pdf (Consultado el 19 de Marzo de 2018)

Engelbrecht, Petra, Nel Mirna, Nel Norma, Nkoli Tlale, y Lloyd Daniel. "Enacting Understanding of Inclusion in Complex Contexts: Classroom Practices of South African Teachers". South African Journal of Education, 1-10. (2015). https://www.researchgate.net/ publication/281403795 Enacting understanding of inclusion in complex contexts Classroom practices_of_South_African_teachers (Consultado el 22 de Marzo de 2018)

Fals-Borda, Orlando. "El problema de cómo investigar la realidad para transformarla por la praxis". Bogotá: Tercer Mundo Editores. (1997).

Figueroa-Céspedes, Ignacio, y Muñoz, Yolanda. "La Guía para la Inclusión Educativa como herramienta de autoevaluación institucional: Reporte de una experiencia". Revista Latinoamericana de Educación Inclusiva, 179-198. (2014). https://www.researchgate.net/ publication/305703121_La_Guia_ para_la_Inclusion_Educativa_como herramienta_de_autoevaluacion institucional_Reporte_de_una_ experiencia (Consultado el 24 de Marzo de 2018)

García-Cedillo, Ismael, Romero-Rubio, Silvia, Flores Barrera, Vasthi Jocabed, y Martínez-Ramírez, Araceli. "Comparación de Prácticas Inclusivas de Docentes de Servicios de Educación Especial y Regular en México". Revista Actualidades Investigativas en Educación, 1-17. (2015). https://www.researchgate.net/ publication/282450778_Comparacion de practicas inclusivas de docentes de servicios de educacion especial y regular en Mexico (Consultado el 08 de Marzo de 2018)

Garnique, Felicita. "Las Representaciones Sociales: Ios Docentes de Educación Básica frente a la Inclusión Escolar". Perfiles Educativos, 99118. (2012). http://www.scielo.org. $\mathrm{mx} /$ scielo.php?script $=$ sci_arttext\& pid $=S 0185-26982012000300007$ (Consultado el 21 de Marzo de 2018) 
Garnique-Castro, Felicita y Gutiérrez-Vidrio, Silvia. "Educación Básica e Inclusión: un Estudio de Representaciones Sociales". Magis. Revista Internacional de Investigación en Educación, 577593. (2012). https://www.redalyc. org/pdf/2810/281022848004.pdf (Consultado el 18 de Marzo de 2018)

Giné-Freixes, Núria; Martí, Elvira; Mentado, Trinidad, y Prats, Mar. "Nuevos Retos para el Profesorado de Secundaria Obligatoria: Formación permanente para la atención a la diversidad en las aulas". Magis Revista Internacional de Investigación en Educación, 67-88. (2009). https://www.researchgate.net/ publication/41126244_Nuevos retos para_el_profesorado_de_secundaria obligatoria formacion permanente para la atencion a la diversidad en las aulas (Consultado el 24 de Marzo de 2018)

Giraldo-Zuluaga, Gloria Amparo. "Ciudadanía: Aprendizaje de una Forma de Vida". Educ. Educ., 76-92. (2015) https://www. redalyc.org/pdf/834/83439194005.pdf (Consultado el 27 de Marzo de 2018)

Gordillo, Enrique; Rivera-Calcina, Renzo: Gamero, Giancarlo. "Conductas Disruptivas en Estudiantes de Escuelas Diferenciadas, Coeducativas e Inter Educativas". Educ. Educ., 427-443. (2014). https://www.redalyc.org/ pdf/834/83433781002.pdf (Consultado el 18 de Marzo de 2018)

Habermas, Juergen y Hoyos Guillermo. "Conocimiento e Interés". Madrid: Taurus. (1984) https://revistas.unal.edu. co/index.php/idval/article/view/29111

Hernández-Sampieri, Roberto, FernándezCollado, Carlos y Baptista-Lucio, Pilar. "Metodología de la investigación". México: Mc Graw Hill. (1998). http:// observatorio.epacartagena.gov.co/wpcontent/uploads/2017/08/metodologia- de-la-investigacion-sexta-edicion. compressed.pdf

Honneth, Axel. "Reificación: Un estudio en la teoría del reconocimiento". Katz, Conocimiento. (2007). https://books. google.com.co/books? id=qEnfXj8 KKfMC\&printsec $=$ frontcover $\& d q=$ reconocimiento $+y+$ reificaci $\% \mathrm{C} 3 \%$ B $3 n \& h l=e s \& s a=X \& v e d=2 a h U K E$ wiQp SzObnrAhUpx1kKHW nCC kQ6AEwAHoECAAQAg\#v=onep age $\& q=$ reconocimiento $\% 20 y \% 20$ reificaci $\%$ C3\%B3n\&f=false

Krichesky, Graciela. "La Construcción de los Procesos de Inclusión/Exclusión en Escuelas Secundarias de Barrios Populares de la Provincia de Buenos Aires". Propuesta Educativa, 101103. (2014). https://www.redalyc. org/pdf/4030/403041713013.pdf (Consultado el 27 de Marzo de 2018)

Llorent-García, Vicente J., y López-Azuaga, Rafael. "Buenas Prácticas Inclusivas en Educación Formal y no Formal: Análisis de Experiencias Educativas reales en la Provincia de Cádiz (España)". Revista de Educación Inclusiva, 174-192. (2013). https://revistaeducacioninclusiva.es/ index.php/REl/article/view/188

Loaiza-Rendón, Carlos Andrés. "La Cultura de la Diversidad: el Telón de Fondo de la Inclusión en la Educación de, con y para todos". Plumilla Educativa, 166-175. (2011). http://revistasum.umanizales. edu.co/ojs/index.php/plumillaeducativa/ article/download/490/585/2495

López, Jose Correia. "Educación Inclusiva "Indicadores de Sentimientos, Actitudes, Preocupaciones y Auto-eficacia de los Profesores". Badajoz, España: (Tesis Doctoral, Universidad de Extremadura, 2014). https://www.semanticscholar. org/paper/Educaci\%C3\%B3n-InclusivaIndicadores-de-Sentimientos\%2C-y-Lo pes/527be0622667bffef26017c41bfda6 
$\underline{\mathrm{b} 59614 \mathrm{a} 8 \mathrm{bd}}$

López, José Manuel. "Atención a la Diversidad y Práctica Educativa en Educación Secundaria Obligatoria: una Contribución a la Identificación de los Estilos de Aprendizaje en Función de las Capacidades y Aptitudes Cognitivas, Motivación, Destrezas de Aprendizaje y Técnicas y H". (Tesis doctoral, Universidad de Murcia, 2013). https://1library.co/document/7qvx1egydiversidad-obligatoria-contribucionidentificacion-aprendizaje-capacidadesmotivacion-aprendizaje.html

Luzuriaga, Lorenzo. Historia de la Educación y la Pedagogía. Buenos Aires: Losada S.A. (1976). https://sociofilosofia.files. wordpress.com/2015/04/historia_de la_educacion_y_de_la_pedagogia_i.pdf Maldonado, María Eugenia, Ortiz-Salazar, María Angélica, y Giraldo-Campuzano, Elsa Ruth. "La Escuela como Contexto Vital, la Nueva Ruralidad: un Pretexto para Incluir-nos". Sophia, 11-20. (2012). https://revistas.ugca.edu.co/index.php/ sophia/article/view/23/36 (Consultado el 23 de Marzo de 2018)

Martínez-Tornay, Antonio. "La Formación Inicial y Permanente del Profesorado para dar Respuesta a la Diversidad. Propuestas y Retos para una Educación Inclusiva de Calidad y Excelencia". Madrid: (Tesis Doctorales TESEO, Universidad Complutense de Madrid, 2014). https://dialnet.unirioja.es/servlet/ tesis?codigo $=97976$

Mehendale, Archana, Mukhopadhyay, Rahul, y Namala, A. "Right to Education and Inclusion in Private Unaided Schools: An Exploratory Study in Bengaluru and Delhi". Economic \& Political Weekly, 4351. (2015). https://www.researchgate. net/publication/283518059_Right_to education and inclusion in private unaided schools An exploratory study in Bengaluru and Delhi

(Consultado el 05 de Abril de 2018)

Montánchez-Torres, Maria Luisa. "Las Actitudes, Conocimientos y Prácticas de los Docentes de la Ciudad de Esmeraldas (Ecuador) ante la Educación Inclusiva. Un Estudio Exploratorio". Valencia, España: Universitat de València. Facultat de Filosofia i Ciències de L'Educació. (2014). https://www.researchgate. net/publication/320044924 Las actitudes conocimientos $y$ practicas de los docentes de la ciudad de_Esmeraldas ante_la_inclusion educativa_un_estudio_exploratorio (Consultado el 29 de Marzo de 2018)

Moreno-Gómez, Diana Lucía, MorenoAbril, Grey Smith, y Amaya-Gelvez, Yolanda. "Prácticas Pedagógicas ¿Responsables e Incluyentes?". Bogotá: Corporación Universitaria Minuto de Dios. (2015) https://ciencia. lasalle.edu.co/cgi/viewcontent. cgi? article $=1233 \&$ context $=a p$ (Consultado el 10 de Abril de 2018)

Morse, Janice. " investigación cualitativa: ¿realidad o fantasía?". En: asuntos críticos de los métodos de investigación cualitativa. 3-27. Antioquia: Facultad de enfermería Universidad de Antioquia. (2003). https://dialnet.unirioja.es/servlet/ articulo?codigo $=5778567$ (Consultado el 13 de Abril de 2018)

Mosquera-Gonzalez, Yorleny. "Imaginarios sobre Educación Inclusiva de Docentes en Instituciones Educativas de Soacha". Horizonte Pedagógico, 22-33. (2011). https://horizontespedagogicos.ibero. edu.co/article/view/98 (Consultado el 15 de Marzo de 2018)

Mundet-Bolós, Anna, Fuentes-Pelaéz, Nuria, y Pastor, Nuria Crescencia. "El fomento de la inclusión social infantil a través de los lenguajes artísticos". Pedagogía social. Revista interuniversitaria 
No. 29. 2017 https://www.redalyc. org/pdf/1350/135049901011.pdf (Consultado el 16 de Marzo de 2018)

Muñoz-Villa, María Loreto, López-Cruz, Mauricio, y Assaél, Jenny. "Concepciones Docentes para responder a la Diversidad: ¿Barreras para la Inclusión Educativa?”. Psicoperspectivas Individuo y Sociedad, 68-79. (2015). https://scielo. conicyt.cl/scielo.php?script=sci artte xt\&pid=S0718-69242015000300007 (Consultado el 21 de Marzo de 2018) Murcia-Peña, Napoleón. “Jóvenes Universitarios y Universitarias: una Condición de Visibilidad aparente en Colombia". Revista Latinoamericana de Ciencias Sociales, Niñez y Juventud, 82-92. (2008). http://revistaumanizales. cinde.org.co/rlcsnj/index.php/RevistaLatinoamericana/article/view/245/129 (Consultado el 03 de Abril de 2018)

Murcia-Peña, Napoleón. "Imaginarios Sociales: Preludios sobre Universidad". Madrid: Editorial Académica Española. (2011).

Murcia-Peña, Napoleón. "La Escuela Como Imaginario Social, "Apuntes para una Escuela Dinámica”. Magistro, 53-70. (2012). https://repository.usta.edu.co/ handle/11634/7512 (Consultado el 16 de Marzo de 2018)

Murcia-Peña, Napoleón, y Jaramillo-Eheverry, Luis Guillermo. "Complementariedad". Armenia: Kinesis. (2008). https:// dialnet.unirioja.es/servlet/ articulo?codigo $=6296692$

Murcia-Peña, Napoleón, y Ospina-Serna, Héctor Fabio. "Regiones investigativas en educación y pedagogía en Colombia: construcción de un mapa de la actividad investigativa de tesis de maestrías y doctorados en el periodo 2000-2010". Manizales: Clnde. (2012). http://www. cinde.org.co/PDF/Regiones $\% 20$ investigativas.pdf
Murcia-Peña, Napoleón, y Murcia-Gómez Jorge Iván. "Perspectivas de investigación en Educación y pedagogía en el eje cafetero". Pedagogía y saberes, 49,55.66. (2018). https://revistas. pedagogica.edu.co/index.php/PYS/ article/view/8170 (Consultado el 08 de Marzo de 2018)

Ohajunwa, Chioma Ogochukwu, y Mckenzie, Judith. "Inclusion of Disability Issues in Teaching and Research in Higher Education". Perspectives in Education, 104-117. (2014). https://www.researchgate.net/ publication/267269802_Inclusion of_disability_issues_in_teaching_ and_research_in_higher_education (Consultado el 07 de Abril de 2018)

Orrego-Noreña, Jhon Fredy, y Portela-Guarín, Henry. "Sentido de la Alteridad en la Formación del Licenciado en Educación Física". Revista Educación Física y Deporte, 49-57. (2009). https://www.researchgate.net/ publication/277844701 Sentido de la_alteridad_en_la_formacion_del licenciado_en_Educacion_Fisica (Consultado el 15 de Abril de 2018)

Ortiz, Iván. "Actitudes de los Estudiantes en Escuelas Segregadas y en Escuelas Inclusivas, hacia la Tolerancia Social y la Convivencia entre Pares". Calidad en la Educación, 68-97. (2016). https://www.researchgate.net/ publication/326944940 Actitudes de los estudiantes en escuelas segregadas y en escuelas inclusivas hacia la tolerancia social_y_la_convivencia_entre_pares (Consultado el 04 de Abril de 2018)

Osorio, Sergio Néstor. "La Teoría Crítica de la Sociedad de la Escuela de Frankfurt "Algunos Presupuestos Teórico Críticos"'. Universidad Militar "Nueva Granada", 104-119. 
(2007). $\quad$ https://www.academia. edu/11472383/LA_TEOR\%C3\%8DA CR\% C 3\%8DTICA_DE_LA SOCIEDAD_DE_LA_ESCUELA DE_FRANKFURT_ALGUNOS PRESUPUESTOS_TE\%C3\%93RICO C R \% C $3 \% \% 8$ D T I C O S Sergio N\%C3\%A9stor Osorio Palabras

Pingle, Sudha, y Garg, Indu. "Effect of Inclusive Education Awareness Programme on Preservice Teachers". The International Academic Forum, 1-23. (2015). https://www.researchgate. net/publication/327940994_Effect of_Inclusive_Education_Awareness Programme_on_Preservice_Teachers (Consultado el 30 de Marzo de 2018)

Pinto, Diana, y Osorio, Belén. "Creación de espacios virtuales para favorecer la atención a la diversidad. Caso: Unidad Educativa Nacional "Padre Mendoza"'. Revista de Investigación, 133-156. (2015). http://ve.scielo. org/scielo.php?script=sci arttext\& pid=S1010-29142015000100007 (Consultado el 03 de Abril de 2018)

Pintos, Juan Luis. "Construyendo realidad(es): los imaginarios sociales. Publicado en realidad". Revista del cono sur de psicología social y política. no. 1. 2001. 7-24. (2001). http://ve.scielo. org/scielo.php?script=sci arttext\& pid $=S 1315-52162005000200003$ (Consultado el 06 de Marzo de 2018)

Pintos, Juan Luis, y Galindo-Arranz, Fermín. "Comunicación Política e Imaginarios Sociales". Revista Comunicación Política en Televisión y Nuevos Medios, Publicado en Salomé Berrocal, (coord.), 111-133. (2003). https://dialnet.unirioja. es/servlet/articulo?codigo $=803551$ (Consultado el 25 de Marzo de 2018)

Plancarte-Casino, Patricia Anabel. "El Índice de Inclusión como herramienta para la mejora escolar". Revista Iberoamericana de Educación, 145-166. (2010). https://red.pucp.edu.pe/ridei/ files/2013/02/130207.pdf (Consultado el 11 de Marzo de 2018)

Ramírez-Iñiguez, Alma Arcelia. "La Educación Escolar como Factor de Inclusión Social desde un planteamiento Sistémico. El Caso de la Niñez jornalera Migrante de los Valles de Culiacán, Sinaloa (México)". Barcelona: (Tesis Doctoral, Facultad de Pedagogía, Universitat de Barcelona, 2014) http://diposit.ub.edu/ dspace/handle/2445/57624

Riquelme-Soto, Verónica, Paz, OrellanaAlonso, Natividad, y Sáez-Serrano, Brenda. "Víctimas invisibles: análisis de la intervención socioeducativa de niñas y niños expuestos a violencia de género en la familia". Pedagogía social. Revista interuniversitaria. no. 34. (2019). https://dialnet.unirioja.es/ servlet/articulo?codigo $=7119151$

Rosa, Olga María, y Villar-Angulo, Luis Miguel. "Inclusión e Interculturalidad. Un Estudio en el Marco de la Enseñanza Universitaria". Revista Nacional e Internacional de Educación Inclusiva, 1229. (2015). https://www.researchgate. net/publication/308937617 Inclusion_e interculturalidad un estudio en el marco de la ensenanza universitaria (Consultado el 19 de Marzo de 2018)

Sanhueza-Henríquez, Susan, GranadaAzcárraga, Maribel, y Bravo-Cóppola, Laura. "Actitudes del Profesorado de Chile y Costa Rica hacia la Inclusión Educativa". Cadernos de Pesquisa, 884-899. (2012). https://www.scielo.br/ pdf/cp/v42n147/13.pdf (Consultado el 03 de Abril de 2018) 
Serres, Michel. "Atlas”. Bogotá, D. C.: Cátedra. (1995).

http://cmap.javeriana.edu.co/servlet/

SBReadResourceServlet?rid= $1264792317343 \_1881472644 \_3387$

Shotter, John. "Realidades Conversacionales". Buenos Aires: Amorrortu Editores. 1993.

Skliar, Carlos. "La Educación (qué es) del otro". Buenos Aires: Noveduc. 2007.

Skliar, Carlos. "Preguntar la Diferencia: Cuestiones sobre la Inclusión". Sophia, 33-43. (2015) https://www.redalyc. org/pdf/4137/413740751004.pdf (Consultado el 23 de Marzo de 2018)

Slee, Roger. "La escuela extraordinaria: Exclusión, escolarización y educación inclusiva". Madrid: EDICIONES MORATA. 2012.

Soto-Builes, Norelly. “¿Diversidad-inclusión vs transformación?". Catedra Abierta. (2007) http://www.revistas.usb.edu.co/ index.php/Agora/article/view/1654

Strauss, Anselm, y Corbin, Juliet. "Bases de la investigación cualitativa. Técnicas y procedimientos para desarrollar la Teoría Fundamentada". Medellín: Universidad de Antioquia. (2002). https://diversidadlocal.files.wordpress. com/2012/09/bases-investigacioncualitativa.pdf

Taylor, Charles. "Los imaginarios sociales modernos”. Barcelona: Paidós. 2006. https://www.uoc.edu/uocpapers/3/dt/ esp/taylor.html

Valenzuela, Blanca Aurelia, Guillén-Lúgigo, Manuela, y Campa-Álvarez, Reyna de los Ángeles. "Recursos para la Inclusión Educativa en el Contexto de Educación Primaria". Infancias Imágenes, 64-75. (2014). https://revistas.udistrital.edu.co/ index.php/infancias/article/view/7436 (Consultado el 02 de Marzo de 2018)

Vásquez-Morales, María Guadalupe, MéndezPineda, Juana María, y MendozaSaucedo, Fernando. "Educación
Inclusiva y Aprendizaje Colaborativo en el Aula: un Estudio de la Práctica Docente Universitaria". Revista Nacional e Internacional de Educación Inclusiva, 171-187. (2015). https:// revistaeducacioninclusiva.es/index.php/ REl/article/view/97 (Consultado el 17 de Marzo 2018)

Vera-Márquez, Ángela Victoria, Palacio-Sanudo, Jorge Enrique, y Patiño-Garzón, Luceli. "Población Infantil Víctima del Conflicto Armado en Colombia Dinámicas de Subjetivación e Inclusión en un Escenario Escolar". Perfiles Educativos, 12-31. (2014) http://www.scielo.org.mx/scielo. php?script=sci_abstract $\&$ pid $=$ S018526982014000300002\&lng =es\&nrm=iso (Consultado el 15 de Abril de 2018)

Vrecer, Natalija, Javrh, Petra, y Capllonch, Marta. "Impacto de la Exclusión Educativa en Ámbitos Sociales". Revista Educación y Pedagogía, 4555. (2010) https://revistas.udea.edu.co/ index.php/revistaeyp/article/view/9819 (Consultado el 21 de Abril de 2018)

Yanez, Deisy. "¿Qué es el enfoque de la investigación? Tipos principales". (2019) https://www.lifeder.com/enfoqueinvestigacion/ (Consultado el 11 de Marzo de 2018) 